\title{
El diálogo interdisciplinario para una ética armónica en la Declaración de Cartagena
}

\author{
Interdisciplinary dialogue for harmonious ethics in the Cartagena Declaration \\ Diálogo interdisciplinar para uma ética harmoniosa na Declaração de Cartagena
}

\author{
Abril García Brito ${ }^{1 *}$, José Mariano Iturbe Sánchez \\ Recibido: 14 de marzo de 2021. Aceptado para publicación: 17 de abril 2021. \\ Publicado en línea: 23 de abril 2021. \\ https://doi.org/10.35454/rncm.v4supl1.291
}

\begin{abstract}
Resumen
En el presente artículo se analizará la Declaración de Cartagena desde una perspectiva filosófica, utilizando como metodología una hermenéutica diatópica, la cual toma los puntos comunes entre la multiplicidad de disciplinas, que permitan una interpretación legítima del ser humano y su nutrición. Es por ello por lo que en esta ocasión se parte del cuarto principio de la Declaración, el cual busca el diálogo interdisciplinario que, desde una interpretación filosófica, solicita no reducir al ser humano a un objeto de estudio o una suerte de especialización temática. Dicho análisis lleva a proponer una ética de la armonía entre las disciplinas.
\end{abstract}

Palabras clave: interdisciplinariedad, diálogo, ética, Declaración de Cartagena.

\section{Summary}

In this article, the Cartagena Declaration will be analyzed from a philosophical perspective, using diatopic hermeneutics as a methodology which consists of taking common points between the multiplicity of disciplines to allow a legitimate interpretation of the human being and its nutrition. For this reason, the point of departure is the fourth principle of the Declaration, which seeks interdisciplinary dialogue to ensure that, from a philosophical interpretation, human beings are not reduced to study objects or some sort of thematic specialization. This analysis leads to propose an ethics of harmony among disciplines.

Keywords: Interdisciplinarity; Dialogue; Ethics; Cartagena Declaration.

\section{Resumo}

O presente artigo, pretende analisar a Declaração de Cartagena desde uma perspectiva filosófica, usando uma hermenêutica diatópica como metodologia, que consiste em tomar pontos comuns entre a multiplicidade de disciplinas, que permitem uma interpretação legítima do ser humano e de sua alimentação. Para isso parte do quarto princípio da Declaração, que busca o diálogo interdisciplinar, que a partir de uma interpretação filosófica busca não reduzir o ser humano a um objeto de estudo ou a uma espécie de especialização temática. Essa análise leva a propor uma ética da harmonia entre as disciplinas.

Palavras chave: interdisciplinaridade, diálogo, ética, Declaração de Cartagena.
Universidad Autónoma del Estado de México, México.

\section{INTRODUCCIÓN}

La ética de la armonía busca servir como propuesta para que las diferentes disciplinas se fecunden unas con otras, a través de un diálogo que mostrará las similitudes de sus resultados y, al mismo tiempo, sus diferencias. Nosotros partimos de que ninguna ciencia o disciplina está diso-
*Correspondencia: Abril García Brito. abrilga2909@gmail.com

ciada, ni puede abarcar ella sola la totalidad del fenómeno humano. De lo contrario, cada ciencia o disciplina hablaría desde su parcialidad, teniendo una visión muy reducida de su objeto de estudio. En este caso, la nutrición clínica ampliaría su horizonte de sentido al tener otras formas de mirar los problemas a los que se enfrenta, como es el caso de la malnutrición, entre otros. 
La búsqueda por generar nuevos diálogos entre las disciplinas surge de una necesidad de nuestro tiempo, ya que cada vez la línea entre las ciencias humanas y las ciencias duras ha de hacerse más tenue. Esto es inminente si se quiere tener una visión más integral del hombre, y así poder subsanar la visión reduccionista con la que cada ciencia aborda al ser humano. De ahí que el presente texto busca ser una reflexión filosófica sobre la Declaración de Cartagena, teniendo como base a la ética como núcleo entre disciplinas.

\section{¿QUÉ PUEDE DECIR LA FILOSOFÍA SOBRE LA DECLARACIÓN DE CARTAGENA?}

La Declaración Internacional sobre el Derecho al Cuidado Nutricional y la Lucha contra la Malnutrición ${ }^{(1)}$ es producto de una asamblea llevada a cabo en Cartagena en 2019. Dicha declaración reconoce el cuidado nutricional como derecho humano, con él se busca combatir la malnutrición relacionada con la enfermedad, y así dar una solución a dicha problemática.

La Declaración consta de 13 principios, que buscan proteger el derecho a la alimentación desde el nivel clínico, y así promover el derecho humano a la nutrición, en tanto que visibiliza a la malnutrición como un problema relacionado con la enfermedad, problema que busca ser subsanado a través de la dignificación del cuidado nutricional, pues este enmarca el derecho a la salud y a la vida digna. Dicho de otro modo, desde una perspectiva filosófica, la Declaración de Cartagena se orienta a ver al ser humano como un ser integral ${ }^{(2)}$, el cual necesita de una alimentación que lo dignifique y constituya, y así hacer frente a la malnutrición, objetivo que se busca alcanzar a través del cuidado nutricional.

El ambiente en el que se gesta esta declaración busca el reconocimiento de una alimentación adecuada para toda persona, y así hacer una lucha contra el hambre. No obstante, es menester matizar que dicha declaración surge en un ambiente clínico, en el cual es de vital importancia dar realce al cuidado nutricional, principalmente a la persona enferma, de ahí que se convierta en una lucha por la malnutrición. Dicho con sencillez, la Declaración de Cartagena tiene sentido en un ambiente clínico, pero ello no debería imposibilitar el diálogo con otras disciplinas, aquí la propuesta de dicho artículo.

La pertinencia de dicha declaración es de vital importancia en tanto que se orienta al cuidado nutricional en pacientes que sufren alguna enfermedad. De ahí que la nutrición clínica encabece la lucha, por ser la que tiene los medios y conocimientos necesarios para hacer frente a este problema, que vincula a la malnutrición y la enfermedad. Sin embargo, la tarea de la nutrición clínica será sesgada si no hay una fecundación entre disciplinas ${ }^{(3)}$. Si bien el cuarto principio de dicha declaración manifiesta un enfoque interdisciplinario en el cuidado nutricional, este solo se reduce a las ciencias de la salud, medicina, enfermería, nutrición y rehabilitación, por mencionar algunas.

\section{CIENCIAS DURAS, CIENCIAS HUMANAS Y CIENCIAS SOCIALES}

Tanto las ciencias duras, como las sociales y humanas se encuentran dentro de un campo limitado de acción, en tenor de que cada una cuenta con sus propios mecanismos para tratar de comprender la realidad. Hasta aquí esto no supone problema alguno, pues ninguna ciencia, de ningún tipo, puede aspirar a un conocimiento completo, pues cada área cuenta con un campo muy específico de estudio, el cual es menester explorar $y$ ahondar en él para conocer ciertas particularidades. No obstante, el problema surge cuando cada ciencia se cierra en sí misma y, como si fuera una mónada, se aísla $\mathrm{y}$ reposa en su propio conocimiento.

Este problema se ha venido asentando en la cultura occidental desde el positivismo, y con él ha venido el auge de las ciencias experimentales. Esas ciencias duras a partir de la prueba y el error dan comprobación a sus teorías, marginan a las ciencias no cuantitativas y a las sociales o humanas, que no pueden experimentar o comprobar sus conocimiento a partir de la prueba y el error, porque en su seno mismo no se puede concebir que el ser humano y su medio se reduzca a pruebas. Más bien tratan de comprender la complejidad del hombre en sus últimas relaciones, entendidas como aquellas que llevan al hombre a buscar la felicidad, la plenitud, la belleza y el bien ${ }^{(4)}$. El ser humano no es prueba y error, tampoco es únicamente un ser social; este es el vínculo entre ambas, y reducirlo a un solo polo es faltar a su aspiración de un ser completo.

\section{NECESIDAD DEL DIÁLOGO INTERDISCIPLINARIO}

La crisis actual, no solo sanitaria, sino también ecológica, nutricional, ética, política y económica, exige un replanteamiento de las ciencias y sus disciplinas. De seguir viendo al hombre desde una ventana particular, no se podrá ver la totalidad; hay que ver a un ser 
humano desde una mirada integral. Cada disciplina se puede abrir en diálogo con otras para alcanzar y realizar esa visión integral, que demuestre que el ser humano es un ser completo, que no se puede explicar dentro de una serie de teorías que comprueben, pero que se queden mudas ante las aspiraciones últimas de este.

Si se logra ver que el ser humano no solo es estadísticas, sino que también es un ser movido por sentimientos, anhelos y preguntas últimas que no se pueden comprobar, entonces se habrá logrado un avance sin precedentes. Urge un diálogo entre disciplinas, no solo entre aquellas pertenecientes a lo que podrían ser un campo determinado, sino entre disciplinas de distinta índole. Hace falta que la física, la biología, la medicina, la enfermería, la nutrición y la terapia física se nutran de las reflexiones filosóficas, antropológicas, sociológicas, históricas y viceversa, las ciencias sociales y humanas tienen que escuchar la voz de las ciencias duras para nutrirse de cada uno de sus aportes.

Dicho lo anterior queda justificada la reflexión filosófica en la Declaración de Cartagena, si se busca dar soluciones reales. Dichas soluciones tienen que emerger de una visión armónica, lo que significa una visión que se cimiente en distintas ópticas de las múltiples disciplinas.

La Declaración de Cartagena reconoce el cuidado nutricional como un derecho humano relacionado con la salud. El problema de fondo, visto desde una perspectiva filosófica en la declaración, es una preocupación inminente por la relación salud-enfermedad, que se manifiesta en la lucha de la nutrición clínica por la malnutrición.

\section{DICOTOMÍAS, FRACTURAS Y UNA POSIBLE VISIÓN INTEGRAL DEL SER HUMANO}

Teniendo a la dicotomía salud/enfermedad como problema de fondo, se ha partir de que las fracturas propuestas por la modernidad, entre un objeto que puede ser analizado y un sujeto que necesariamente analiza al objeto, terminaron por hacer del ser humano un objeto de estudio que no dista de un objeto cualquiera. Dicho con sencillez, con la creciente racionalización del mundo a partir del positivismo, el occidente moderno optó por agudizar una visión del mundo fragmentada, y con él a un ser humano igualmente dividido.

Si las disciplinas se han dividido, también se ha fracturado una visión completa del hombre y de la realidad. Una disciplina que solo toma al hombre como un fragmento dará un conocimiento igual de fragmentario de este $y$, por ende, las problemáticas que se generen de una visión sesgada no podrán resolverse más que de manera incompleta ${ }^{(5)}$.

En el caso de la Declaración de Cartagena, el problema salud-enfermedad que debe afrontar la nutrición clínica, con vistas a combatir la malnutrición a partir del cuidado nutricional, debe ser abordado no solo como un problema que compete a la nutrición, medicina, enfermería y de más disciplinas de la salud, pues estas, en soledad, solo pueden dar interpretaciones sesgadas correspondientes a su área de trabajo, profundizando únicamente en una visión fracturada del ser humano. Se ha de tener disposición de abrir el diálogo con las humanidades y las disciplinas sociales, por ejemplo, con la filosofía, la cual le puede brindar a las ciencias de la salud, la posibilidad de mirar al hombre desde una nueva perspectiva, en la que el ser humano no es visto como un objeto que debe ser sanado; mas bien, ver al ser humano como un ser completo, que necesita una mirada armónica entre las disciplinas para tener una visión integral del hombre.

Filosóficamente, si se quiere comprender algún problema relacionado con el hombre o con su naturaleza, se ha de partir de la pregunta ¿Qué es el hombre? ${ }^{(6)}$ Este cuestionamiento, más que resultar superfluo para las ciencias duras, representa el trasfondo de cualquier problemática que les concierne, pues el hombre es el eje sobre el cual ha de girar cualquier conocimiento, es decir, si la nutrición es la encargada de mantener el equilibrio entre los nutrientes contenidos en los alimentos para el correcto funcionamiento de las funciones vitales, dicho funcionamiento es estudiado a partir de que es aplicado al hombre. Si la medicina es el ejercicio por el cual se busca curar cierta enfermedad, dicha enfermedad gira entorno a la naturaleza humana, a su vez, la filosofía se ha de encargar de estudiar las cuestiones primeras y últimas del hombre, esto por citar tres ejemplos de la vasta variedad de disciplinas. En todos ellos el núcleo es el hombre, si no se tiene en cuenta esto, se caerá en una suerte de fragmentación del conocimiento, en el que se pierde el corazón del problema, que en términos de antropología filosófica es el bienestar del hombre.

De esta forma, lo que se propone no es centrarse en la especialización, hecho que es legítimo, pero no fundamental, de lo que se trata es centrarse en el problema, para así lograr un diálogo entre las disciplinas. No se trata de que cada disciplina o ciencia hable desde su propia especialización, sino que dialoguen teniendo un problema común, en este caso saber ¿Qué es hombre? Para así poder llevar este problema a puntos específicos 
como el de salud-enfermedad, sin que ello signifique renunciar al núcleo del problema.

A través de intentar dar respuesta a la pregunta por el hombre se puede descubrir que este no es un individuo, un ser aislado, arrojado al mundo para conquistarlo. Pensar que el hombre es un ser sin aspiración a la plenitud, a lo trascendente o a la salvación es una visión enajenante y fatalista, el hombre no puede ser un ser dividido o fragmentado. En cambio, si se responde que el hombre es un ser en relación, y corresponde más a una interpretación personalista, entonces se puede cumplir con una visión no tan reduccionista, que permita que el ser humano se desarrolle en comunión con los otros y su entorno, y tener plena conciencia de que lo que el hombre en verdad busca no es satisfacer fines utilitaristas, sino alcanzar la plenitud.

Enfocada a la Declaración de Cartagena, el diálogo interdisciplinario permitirá hacer consciente al personal de salud de que el destino final de la persona no está restringido a su estado de salud, entendido como el correcto funcionamiento psicosomático de un organismo, sino, quizás, a alcanzar su plenitud, es decir, la correcta armonía entre el cuerpo, el intelecto y el espíritu.

\section{LA SALUD: UNA TAREA INTERDISCIPLINARIA}

Si se atiende al término salud, este proviene del latín salu, que quiere decir salvación, salud, saludar, seguridad. En francés, salut significa estar a salvo; mientras que en alemán heil significa salvación y salud; al igual que en español, "salud" quiere decir salud o acción de saludar. Tomando como ejemplo algunas de las raíces citadas anteriormente, se deducen que la salud tiene una significación salvífica, es decir, está relacionada con la salvación. Si "salud" quiere decir salvado, su contraposición sería "enfermo", que significaría un estado de malsano, entonces se deduce que las disciplinas de la salud lo que buscan es la salvación del ser humano ${ }^{(5)}$. Ahora bien, una definición más contemporánea del término salud es la que propone la Organización Mundial de la Salud (OMS) como: «La salud es un estado de completo bienestar físico, mental y social, y no solamente la ausencia de afecciones o enfermedades ».

En este sentido, una definición que atienda al sentido original puede contribuir a plantear los alcances y deficiencias de una definición enmarcada en el ámbito de un conjunto de ciencias determinadas. En este caso, atender al sentido primero del término salud puede ayudar a comprender mejor el concepto vigente dado por la OMS, lo cual propiciaría un significado que puede ser analizado y enriquecido por diferentes disciplinas. De esta forma, retornar al significado primero del término salud puede contribuir al entendimiento de una definición más completa del mismo, y así comprender que la salud puede ser un concepto interdisciplinario, en tanto que puede orientar hacia la comprensión del ser humano como un ser integral, no escindido.

Es preciso matizar que de la expresión "salvar al hombre", el término "salvación" significa un estado completo de llevar al hombre a lo completo, a la plenitud, tarea que es imposible para una sola disciplina. El hombre es una unidad, un ser integral, al cual es imposible salvar desde la fractura. No se puede llegar a la salvación si el hombre está dividido en muchas especialidades.

El diálogo entre filosofía y disciplinas de la salud es una necesidad imperante de nuestro tiempo, si es que se quiere superar la especialización reinante en la actualidad. No se trata de suponer que el exclusivismo de cada disciplina ha sido fatal, ya que la especialización ayuda a comprender un problema de forma más detallada, el problema es que esta ha traído consigo un modo tan específico que no se puede aplicar a ninguna otra cosa.

La interdisciplinariedad ha de ser la encargada de superar el problema de la especialización, a través de la ética de la armonía. La nutrición, la medicina, la enfermería, y en general, todas las disciplinas de la salud han de aprender de la filosofía a tomar consciencia de que el hombre es más que un objeto de estudio, que puede ser "medido" y "sanado" a partir de medicamentos. Por otro lado, la filosofía necesita aprender de las ciencias de salud acerca del funcionamiento cuantitativo del hombre. Las disciplinas deben estar en relación armónica, a través de una ética que concientice a los hombres de que su estado en el mundo implica una parte corporal, otra intelectiva y otra espiritual, para así tener una salud equilibrada. Solo en el trabajo constante de filósofos, médicos, nutriólogos, enfermeros y sociólogos, el ideal que tiene la Declaración de Cartagena será realidad.

\section{CONCLUSIÓN}

La filosofía encuentra una problemática y una posibilidad de diálogo en la Declaración de Cartagena. La problemática radica en que la estructura, desde la cual está escrita, promueve un replanteamiento de los paradigmas de comprensión social, humana, de salud y nutricional, que se puede abordar desde la filosofía, dejando ver la multiplicidad de la cual está constituido el ser humano.

La distinción imperante entre las disciplinas sociales, humanas y duras hace que cada una de ellas observe 
un fenómeno determinado desde su particularidad. Los descubrimientos que tuvieron cada una fueron relevantes en una época en la que la especialización cobró importancia. Sin embargo, al analizar un objeto específico desde sus propios métodos, no lograron observar sus límites y sus alcances, problema que solo se subsanará en el diálogo interdisciplinario.

El diálogo interdisciplinario es una necesidad de nuestro tiempo, las disciplinas no pueden seguir encerradas en su propio núcleo, pues los problemas imperantes exigen una visión holista de la realidad, y mientras cada disciplina no se abra a mirar nuevos horizontes que nutran sus perspectivas, las dificultades actuales no se podrán subsanar.

Solo en la apertura hacia una visión armónica del ser humano, las fracturas y las divisiones con las cuales las disciplinas estudian al hombre se podrán superar. Si el ser humano sigue viéndose desde la estrechez de una disciplina, nunca podrá aspirar a tener una visión integral de sí mismo y, por ende, no podrá aspirar a la trascendencia.

El diálogo que posibilita la Declaración de Cartagena es un terreno fértil para la fecundación entre disciplinas, por ser una problemática de alcance universal, que necesita ser atendida de manera inmediata. La filosofía puede contribuir en el logro de sus metas a partir de concientizar al personal de salud y nutrición a través de una ética que permita una visión integral del ser humano, una ética de la armonía entre disciplinas.

\section{PUNTOS CLAVE}

- Filosofía ante la Declaración de Cartagena

- Necesidad de la interdisciplinariedad en la Declaración de Cartagena

- Búsqueda de una visión integral del ser humano

- Cambio de conciencia a partir de una ética de la armonía.

\section{Financiación}

El presente estudio no tuvo financiación.

\section{Conflicto de intereses}

Los autores declaran no tener conflicto de intereses.

\section{Declaración de autoría}

AGB y JMIS contribuyeron igualmente a la concepción y diseño de la investigación; AGB participó en el diseño de la investigación; JMIS contribuyó a la adquisición y análisis de los datos; AGB y JMIS aportaron en la interpretación de los datos; y AGB y JMIS redactaron el manuscrito. Los autores revisaron el manuscrito, acuerdan ser plenamente responsables de garantizar la integridad y precisión del trabajo, y leyeron y aprobaron el manuscrito final.

\section{Referencias bibliográficas}

1. Cárdenas D, Bermúdez $\mathrm{CH}$, Echeverri S, Pérez A, Puentes M, López M, et al. Declaración de Cartagena. Declaración Internacional sobre el Derecho al Cuidado Nutricional y la Lucha contra la Malnutrición. Nutr Hosp. 2019;36(4):97498. doi: 10.20960/nh.02701.

2. Eliade M (editor). La búsqueda. $3^{\mathrm{a}}$ edición. Barcelona: Kairós. 2008.

3. Panikkar R (editor). Las puertas estrechas del conocimiento. Barcelona: Herder. 2009.

4. Dilthey W (editor). Introducción a las ciencias del espíritu. México: FCE. 2015.

5. Panikkar R (editor). La religión, el mundo y el cuerpo. Barcelona: Herder. 2014.

6. Panikkar R (editor). La plenitud del hombre. Madrid: Siruela. 1999. 